\title{
Recognizable Clinical Subtypes of Obstructive Sleep Apnea After Ischemic Stroke: A Cluster Analysis
}

\author{
Chung-Yao Chen (iD) ${ }^{1,2}$ \\ Chia-Ling Chen ${ }^{3,4}$
}

'Department of Physical Medicine and Rehabilitation, Chang Gung Memorial Hospital, Keelung, Taiwan; ${ }^{2} \mathrm{~S}$ chool of Medicine, College of Medicine, Chang Gung University, Taoyuan, Taiwan; ${ }^{3}$ Department of Physical Medicine and Rehabilitation, Chang Gung Memorial Hospital, Linkou, Taiwan; ${ }^{4}$ Graduate Institute of Early Intervention, College of Medicine, Chang Gung University, Taoyuan, Taiwan
Correspondence: Chung-Yao Chen Department of Physical Medicine and Rehabilitation, Chang Gung Memorial Hospital, No. 200, Ln. 208, Jijin Ist Road, Anle Dist., Keelung City, 204, Taiwan

Tel +886-2-2432-9355 Ext 2545

Fax +886-2-2431593I

Email jongyau2002@gmail.com
This article was published in the following Dove Press journal: Nature and Science of Sleep

Background and Purpose: Obstructive sleep apnea (OSA) increases risk of stroke recurrence and mortality in ischemic stroke patients. However, equivocal treatment effects warrant further categorization of post-stroke OSA for risk stratification and individualized treatment planning.

Methods: The study recruited 232 ischemic stroke patients with moderate-to-severe OSA admitted for inpatient rehabilitation consecutively. Latent class analysis was performed based on sex, age, smoking, daytime sleepiness, depression, obesity, sedative use, atrial fibrillation, diabetes, dyslipidemia, hypertension, recurrent stroke and dysphagia. The augmentation index, a marker of arterial stiffness, was measured by applanation tonometry.

Results: A three-cluster model provided the best fit. Cluster $1(\mathrm{n}=84,36.2 \%)$ was older in age, predominantly female, with the highest hypopnea index and prevalence of atrial fibrillation. Moreover, patients in Cluster 1 had significantly higher augmentation index than those in Cluster 2. Cluster 2 patients $(\mathrm{N}=80,34.5 \%)$ were of older age, predominantly male, with the highest prevalence of depression, the lowest prevalence of hypertension and had the most normal body mass index (BMI). Additionally, Cluster 2 had less nocturnal hypoxia as compared to Cluster 3 . Cluster $3(n=68,29.3 \%)$ was the youngest in age, predominantly male, with the highest BMI, cumulative risk score, and prevalence of dyslipidemia of the three clusters.

Conclusion: Post-stroke OSA can be categorized into three clinical phenotypes. Patients in Clusters 1 and 3 both had elevated cardiovascular risk and treatment can be based on their distinct characteristics. Patients in Cluster 2 had relatively lower risk of cardiovascular events and the benefits of OSA treatment requires further study.

Keywords: obstructive sleep apnea, ischemic stroke, cluster analysis, latent class analysis

\section{Introduction}

Severe obstructive sleep apnea (OSA) doubles the risk of incident stroke and is a risk factor for stroke recurrence and mortality, but can be improved by continuous positive airway pressure treatment. ${ }^{1}$ OSA is highly prevalent in stroke patients but compliance with standard continuous positive airway pressure treatment is poor. Consequently, the effectiveness of continuous positive airway pressure treatment for reducing stroke risk is equivocal. ${ }^{1}$

Previous cluster analysis identified three distinct clusters: a "disturbed sleep group," a "minimally symptomatic group," and an "excessive daytime sleepiness (EDS) group," using the Icelandic Sleep Apnea Cohort based on latent class 
analysis (LCA) using clinical symptoms and comorbidities. $^{2}$ Keenan et al confirmed and extended these results by recruiting international participants from the Sleep Apnea Global Interdisciplinary Consortium. ${ }^{3}$ However, both these analyses were mainly performed on middle-aged patients with obesity. A study by Mazzotti et al using the Sleep Heart Health Study (SHHS) cohort with mean age of $66.0 \pm 10.5 \mathrm{y} / \mathrm{o}$ and body mass index (BMI) of $30.4 \pm 5.7 \mathrm{~kg} / \mathrm{m}^{2}$, identified four clusters, including the three previously mentioned clusters plus a moderate sleepy subtype; the excessively sleepy subtype had the highest apnea-hypopnea index and increased prevalence and incidence of cardiovascular events compared to the three other clusters. ${ }^{4}$ Therefore, phenotypes found by cluster analysis may have prognostic value and characteristics suitable for precision medicine. However, the typical clinical manifestations of OSA, such as daytime sleepiness, are not obvious in stroke patients. ${ }^{5}$ Moreover, more than $50 \%$ of ischemic stroke patients reported insomnia ${ }^{6}$ which could result from depression, anxiety, brain damage, sleep-disordered breathing, medications, and environmental disturbances (eg, in a rehabilitation ward). ${ }^{7}$ Therefore, it is questionable whether previously mentioned symptom-based subtypes of OSA can be applied to patients with ischemic stroke. Schutz et al conducted cluster analysis of ischemic stroke patients with OSA diagnosed by home sleep apnea testing based on symptoms, comorbidities and OSA severity index and identified three clusters that differed by age, stroke severity and OSA severity. ${ }^{8}$ However, the major determinants found in their study, age, stroke severity and OSA severity, are well-known risk factors for further cardiovascular events and thus fail to provide additional useful information for the management of OSA in ischemic stroke patients.

This study was designed to identify subgroups of moderate-to-severe OSA patients with comorbid ischemic stroke using LCA based on symptoms and comorbidities instead of OSA severity, in order to improve the management of OSA in stroke patients. The study also examined whether patient subgroups differed in demographic, polysomnographic and vascular characteristics.

\section{Methods}

\section{Participants}

This study enrolled consecutive post-acute ischemic stroke patients with OSA who were admitted for rehabilitation at a teaching hospital. The exclusion criteria were as follows: severely impaired consciousness; unstable medical or neurological status such as active infection or recent transient ischemic attack; previous history of intracranial hemorrhage or malignance; and chronic kidney disease at stage 3 or higher. Participants with other sleep disordered breathing, such as central sleep apnea syndrome or central hypoventilation, were also excluded.

The study was conducted in accordance with the Declaration of Helsinki. The institutional review board of Chang Gung Medical Foundation approved the study protocol (IRB number: 201802080B0). Informed consent was given by participants and/or their legal guardians if participants had certain degree of cognitive or communicative impairments due to stroke.

\section{Clinical Evaluation}

The researchers collected demographic data and a thorough history of stroke risk factors. We considered smoking (active smokers vs nonsmokers; ex-smokers were considered nonsmokers if they had stopped smoking more than three months prior to stroke onset), hypertension (HTN, history of HTN, or systolic blood pressure $>140 \mathrm{mmHg}$ or diastolic blood pressure $>90 \mathrm{mmHg}$ before or two weeks after stroke onset), and diabetes mellitus (history of diabetes mellitus or fasting blood glucose $>126 \mathrm{mg} / \mathrm{dL}$ ). Dyslipidemia was defined based on current lipid levels or taking anti-dyslipidemia medications in the past two weeks. The cut-off values were $240 \mathrm{mg} / \mathrm{dL}, 160 \mathrm{mg} / \mathrm{dL}, 40 \mathrm{mg} / \mathrm{dL}$ and $200 \mathrm{mg} / \mathrm{dL}$ for higher total cholesterol, higher low-density lipoprotein, lower high-density lipoprotein, and higher triglycerides, respectively. ${ }^{9}$ A speech-language pathologist confirmed the diagnosis of dysphagia. Cardiac arrhythmia was assessed by 12-lead electrocardiogram or 24-hour Holter electrocardiogram if stroke etiology was uncertain after the initial evaluation. Neck circumference, BMI, Epworth Sleepiness Scale ${ }^{10}$ and the Barthel Index $(\mathrm{BI})^{11}$ were measured on the same day as polysomnography examination. We used the BI to evaluate activities of daily living and functional outcomes and to represent stroke severity. The EDS is associated with increased incident cardiovascular morbidity and mortality $^{12,13}$ and was defined as an Epworth Sleepiness Scale $\geq 10 .{ }^{10}$ We categorized underweight as BMI $<18.5 \mathrm{~kg} / \mathrm{m}^{2}$, overweight as $\mathrm{BMI} \geq 24.0$ to $<27.0 \mathrm{~kg} / \mathrm{m}^{2}$, and obesity as BMI $\geq 27.0 \mathrm{~kg} / \mathrm{m}^{2}$ according to the definitions of the Health Promotion Administration 
of Taiwan. Depression was assessed with the Patient Health Questionnaire- $9^{14}$ and defined by a Patient Health Questionnaire-9 score $\geq 10$, with a sensitivity of $0.80(95 \%$ CI, $0.62-0.98)$ and a specificity of $0.78(95 \%$ CI, 0.69-0.83). ${ }^{15}$ The cumulative risk score was defined as the sum of the presence of major cardiovascular risk factors, including smoking, obesity, HTN, diabetes mellitus and dyslipidemia. ${ }^{16}$

\section{Polysomnography}

The polysomnography was conducted at the sleep center using a Compumedics Profusion system (Australia). At least six hours of recorded time was required to obtain a valid measurement. We used the American Academy of Sleep Medicine (AASM) scoring manual version 2.0.3 to diagnose sleep apnea ${ }^{17}$ and calculated the oxyhemoglobin desaturation index, defined as the average number of desaturation episodes per hour. Apnea was defined as the discontinuation of airflow for $\geq 10$ seconds; hypopnea was defined as a reduction of $>30 \%$ in airflow for $>10$ seconds with either oxygen desaturation $\geq 3 \%$ or an arousal. Diagnosis of OSA was made when $>50 \%$ of respiratory events were of obstructive or of mixed types. Central sleep apnea syndrome was diagnosed when $\geq$ $50 \%$ of respiratory events were of central type. We defined moderate-to-severe OSA as $>15$ apneas and/or hypopneas per sleep hour (apnea-hypopnea index $>15$ events $\left.\cdot h^{-1}\right)$.

\section{Arterial Stiffness}

Detailed methods to evaluate arterial stiffness by pulse-wave analysis using the SphygmoCor ${ }^{\circledR}$ CPV System (AtCor Medical, Sydney, Australia) have been described elsewhere ${ }^{18,19}$ and in our previous work..$^{20}$ Briefly, measurements were taken immediately after the patients woke up and before breakfast. For pulse wave calibration, blood pressure was recorded five minutes before vascular measurements over the non-paretic arm. The applanation probe was placed on the radial artery of the non-paretic arm. The ascending aortic waveform was generated from the radial signal using SphygmoCor ${ }^{\circledR}$ software (version 9.0) ${ }^{21}$ and the augmentation index (AIx) as a measure of systemic arterial stiffness ${ }^{22}$ was computed as the difference between the second and first systolic peaks divided by the central pulse pressure. Given that the AIx is associated with heart rate, we used an index corrected for a heart rate of 75 bpm (AIx@75). ${ }^{23}$

\section{Statistics}

Cluster analysis among ischemic stroke patients with previously untreated moderate-to-severe OSA was performed using LCA with 13 clinically relevant variables, including sex, old age (age $\geq 65$ vs $<65$ ), cigarette smoking, EDS (Epworth Sleepiness Scale $\geq 10$ vs $<$ 10), depression (Patient Health Questionnaire-9 score $\geq$ 10 vs $<10$ ), obesity (BMI $\geq 27,24 \sim 27,18.5 \sim 24$, < $18.5 \mathrm{~kg} / \mathrm{m}^{2}$ ), sleep disturbance with sedative use, including hypnotics and sedative antidepressants, comorbidities including atrial fibrillation (Af), diabetes mellitus, dyslipidemia, HTN, recurrent stroke and dysphagia. Models with between two and six clusters were compared and the optimal number of clusters was determined based on meaningful clinical interpretability and parsimony according to the Bayesian information classification indices, a nonsignificant $X^{2}$ statistic with $P>0.05$ (using the bootstrap $\mathrm{L}^{2}$ value), as well as the bootstrap likelihood ratio test to statistically test the different cluster models. Lower Bayesian information classification values represent better model fit. Once the optimal clustering solution was determined, the LCA calculated the probability of participants being part of each constructed class and assigned each participant membership to the class considering the highest probability. Then we summarized and compared clinical symptoms, demographic characteristics, parameters of OSA and arterial stiffness across classes using the following methods: 1. For continuous and normally distributed variables: analysis of variance followed by post hoc Scheffe's analyses for homogeneous variances or the Welch test followed by post hoc Games-Howell analyses for heterogeneous variances; 2. For continuous and non-normally distributed variables: Kruskal-Wallis one-way analysis of variance followed by post hoc Dunn's multiple-comparison test; $3 . X^{2}$ or Fisher's exact tests for categorical variables. When group differences were observed with $X^{2}$ or Fisher's exact tests $(P<0.05)$, we performed between-class comparisons to understand which between-class differences were driving overall associations. Given three pairwise comparisons, a Bonferroni-corrected value of $P<0.0167$ was considered a statistically significant between-class difference. Latent Gold 5.0 (Vermunt \& Magidson, 2005) and SPSS (IBM SPSS Statistics 25, SPSS Inc., Chicago, IL) were used. 


\section{Results}

The study included 232 ischemic stroke patients with moderate-to-severe OSA. On average, the median age was 63.7 years (interquartile range (IQR) 56.7-73.5 years), $69.8 \%$ were male, median BMI was $24.3 \mathrm{~kg} / \mathrm{m}^{2}$ (IQR 21.8-26.7 kg/m²) and the median BI was 40 (IQR 25-50) due to ischemic stroke. The median duration between stroke onset and polysomnography study was 2.7 months (IQR 1.2-4.8 months). The sample characteristics are summarized in Table 1. Table 2 shows the initial LCA results of two- to six-cluster models. Examining these statistics, the two-cluster model seemed the best fit as it had the lowest Bayesian information classification value. Five-cluster and sixcluster models had bootstrap $P<0.05$. The results from the bootstrap likelihood ratio test indicated that the three-cluster model was better than the two-cluster model ( $-2 \mathrm{LL}$ Diff $=41.84, P=0.032)$. Thus, we selected the three-cluster model as the most appropriate model. The characteristics of the three clusters are described below and summarized in Table 1. The markers of OSA severity, including apnea-hypopnea index and oxyhemoglobin desaturation index, did not differ significantly among the three clusters. Also, the three clusters did not differ significantly in the frequency of sedative usage and the prevalence of EDS.

Table I Demographic, Clinical Characteristics, OSA and Arterial Stiffness Parameters of the Total Cohort and by Clusters

\begin{tabular}{|c|c|c|c|c|c|}
\hline & Overall Cohort & Cluster I & Cluster 2 & Cluster 3 & $\mathbf{p}^{*}$ \\
\hline $\mathrm{N}(\%)$ & $232(100 \%)$ & $84(36.2 \%)$ & $80(34.5 \%)$ & 68 (29.3\%) & \\
\hline \multicolumn{6}{|l|}{ Clinical variables } \\
\hline Age $(y / o)$ & $63.7(56.7-73.5)$ & $70.3(60.4-74.7)^{\ddagger}$ & $70.5(59.7-76.4)^{\S}$ & $57.8(52.2-62.2)^{\ddagger}, \S$ & $<0.001$ \\
\hline Male (n) & $162(69.8 \%)$ & $18(21.4 \%)^{\dagger, ~} \neq$ & $77(96.2 \%)^{\dagger}$ & $67(98.5 \%)^{\ddagger}$ & $<0.001$ \\
\hline BMI $\left(\mathrm{kg} / \mathrm{m}^{2}\right)$ & $24.4 \pm 4.1$ & $24.4 \pm 5.3^{\dagger, \neq}$ & $22.8 \pm 2.6^{\dagger .} \S$ & $26.2 \pm 2.9^{\ddagger} \S$ & $<0.001$ \\
\hline Smoking (n) & 89 (38.4\%) & $0^{\dagger,} \neq$ & $42(52.5 \%)^{\dagger}$ & $47(69.1 \%)^{\ddagger}$ & $<0.001$ \\
\hline EDS (n) & II8 (50.9\%) & 44 (52.4\%) & 38 (47.5\%) & $36(52.9 \%)$ & 0.757 \\
\hline Depression (n) & $93(40.1 \%)$ & $27(32.1 \%)^{\dagger}$ & $45(56.2 \%)^{\dagger, ~ \S}$ & $21(30.9 \%)^{\S}$ & 0.001 \\
\hline Af $(n)$ & $44(19.0 \%)$ & $27(32.1 \%)^{\dagger, ~ \neq}$ & $8(10.0 \%)^{\dagger}$ & $9(13.2 \%)^{\ddagger}$ & 0.001 \\
\hline $\mathrm{DM}(\mathrm{n})$ & 98 (42.2\%) & $40(47.6 \%)$ & $26(32.5 \%)$ & 32 (47.1\%) & 0.093 \\
\hline Dyslipidemia (n) & I 78 (76.7\%) & $62(73.8 \%)^{\ddagger}$ & $48(60.0 \%)^{\S}$ & $68(100.0 \%)^{\S, ~} \neq$ & $<0.001$ \\
\hline Hypertension (n) & $202(87.1 \%)$ & $80(95.2 \%)^{\dagger}$ & $55(68.8 \%)^{\dagger, \S}$ & $67(98.5 \%)^{\S}$ & $<0.001$ \\
\hline Recurrent stroke $(n)$ & $70(30.2 \%)$ & 29 (34.5\%) & $16(20.0 \%)$ & $25(36.8 \%)$ & 0.048 \\
\hline Dysphagia (n) & $53(22.8 \%)$ & $24(28.6 \%)^{\ddagger}$ & $29(36.2 \%)^{\S}$ & $0^{\ddagger}$ § & $<0.001$ \\
\hline Barthel index & $40.0(25.0-50.0)$ & $30.0(21.3-45.0)^{\ddagger}$ & $32.5(20.0-48.8)^{\S}$ & $45.0(40.0-53.8)^{\ddagger}, \S$ & $<0.001$ \\
\hline Neck circumference $(\mathrm{cm})$ & $38.0(35.5-40.0)$ & $35.0(33.1-38.0)^{\dagger, \ddagger}$ & $38.0(37.0-39.0)^{\dagger, \S}$ & $40.0(38.6-42.0)^{\ddagger}, \S$ & $<0.001$ \\
\hline Cumulative risk score & $3.0(2.0-3.0)$ & $2.5(2.0-3.0)^{\ddagger}$ & $2.0(1.0-3.0)^{\S}$ & $4.0(3.0-4.0)^{\ddagger . \S}$ & $<0.001$ \\
\hline Sedatives usage $(n)$ & $98(42.2 \%)$ & $36(42.9 \%)$ & $32(40.0 \%)$ & $30(44.1 \%)$ & 0.871 \\
\hline \multicolumn{6}{|l|}{ OSA parameters } \\
\hline $\mathrm{AHI}$ (events/hour) & $40.5(27.7-56.6)$ & $44.9(27.6-55.4)$ & $37.8(25.5-55.3)$ & $42.5(30.4-58.2)$ & 0.428 \\
\hline ODI (events/hour) & $34.4(20.3-55.6)$ & $40.1(20.8-56.1)$ & $28.1(19.6-57.6)$ & $35.9(19.7-54.4)$ & 0.649 \\
\hline Apnea index (events/hour) & $15.5(6.5-40.5)$ & $14.6(5.1-33.7)$ & $14.6(7.7-44.1)$ & $17.3(6.3-43.2)$ & 0.240 \\
\hline OA index (events/hour) & $10.0(4.5-20.4)$ & $9.5(2.9-18.3)$ & $9.7(5.4-18.7)$ & I3.I (4.8-28.2) & 0.232 \\
\hline CA index (events/hour) & $0.4(0-1.9)$ & $0.3(0-1.5)$ & $0.8(0-2.4)$ & $0.5(0-1.3)$ & 0.094 \\
\hline MA index (events/hour) & $1.2(0-7.2)$ & $1.0(0-8.8)$ & $1.0(0-7.3)$ & $1.4(0-6.4)$ & 0.874 \\
\hline Hypopnea index (events/hour) & $19.3(12.2-26.0)$ & $22.7(|4.6-3| .0)^{\dagger, \ddagger}$ & $15.2(9.1-23.9)^{\dagger}$ & $19.2(12.5-23.0)^{\ddagger}$ & 0.001 \\
\hline Mean $\mathrm{SpO}_{2}(\%)$ & $93.0(91.6-94.6)$ & $93.0(91.4-94.0)$ & $93.0(91.7-94.7)$ & $93.8(92.4-95.0)$ & 0.054 \\
\hline Minimum $\mathrm{SpO}_{2}(\%)$ & $77.0(65.0-82.8)$ & $76.0(66.0-82.0)$ & $79.0(69.3-83.0)^{\S}$ & $75.0(57.0-81.0)^{\S}$ & 0.039 \\
\hline Sleep efficiency (\%) & $71.3(60.0-80.9)$ & $71.4(57.0-80.9)$ & $68.5(56.2-76.7)^{\S}$ & $74.6(65.2-86.1)^{\S}$ & 0.007 \\
\hline Alx@75 (\%) & $27.8 \pm 8.8$ & $30.4 \pm 9.0^{\dagger}$ & $26.0 \pm 9.4^{\dagger}$ & $27.0 \pm 6.9$ & 0.007 \\
\hline
\end{tabular}

Notes: Values are means \pm standard deviation or medians (interquartile ranges) unless otherwise stated. *P value from analysis of variance and chi-square or Fisher exact tests across 3 clusters. ${ }^{\dagger}$ Cluster I vs 2 comparisons significant. ${ }^{\ddagger}$ Cluster I vs 3 comparisons significant. ${ }^{\S}$ Cluster 2 vs 3 comparisons significant.

Abbreviations: BMI, body mass index; EDS, excessive daytime sleepiness; Af, atrial fibrillation; DM, diabetic mellitus; AHI, apnea-hypopnea index; ODI, oxyhemoglobin desaturation index; OSA, obstructive sleep apnea; OA, obstructive apnea; CA, central apnea; MA, mixed apnea; SpO2, oxyhemoglobin saturation by pulse oximetry; Alx@75, augmentation index adjusted for heart rate at 75 beats per minute. 
Table 2 Model-Fit Statistics for Latent Class Analysis Results

\begin{tabular}{|l|c|c|c|}
\hline Number of Clusters & BIC & LL & p value* \\
\hline 2 & 4130.2121 & -1975.235 & 0.188 \\
3 & 4180.9677 & -1954.315 & 0.090 \\
4 & 4230.2667 & -1932.668 & 0.064 \\
5 & 4295.2216 & -1918.848 & 0.026 \\
6 & 4363.6787 & -1906.779 & 0.020 \\
\hline
\end{tabular}

Note: $* X^{2}$ statistic using the bootstrap $L 2$ value.

Abbreviations: BIC, Bayesian information criteria; LL, log likelihood.

Cluster 1 was older in age, predominantly female (78.6\%) and had the highest prevalence of Af and the lowest rate of smoking of the three clusters. Although the severity of OSA did not differ among the three clusters, the hypopnea index was highest in Cluster 1. In addition, patients in Cluster 1 had significantly higher AIx@75 than those in Cluster 2.

Cluster 2 was older in age, predominantly male (96.3\%) and had the highest prevalence of depression, the lowest prevalence of HTN, and the most normal BMI. Although the prevalence of recurrent stroke differed significantly among the three clusters $(\mathrm{P}=0.048)$, post-hoc analysis only revealed a trend that patients in Cluster 2 had lower prevalence of recurrent stroke that those in Clusters 1 and 3. Additionally, Cluster 2 had a higher level of minimum oxyhemoglobin saturation by pulse oximetry and lower sleep efficiency compared to Cluster 3.

Cluster 3 was predominantly male $(98.5 \%)$, the youngest in age, and had the highest BMI, BI, neck circumference, prevalence of dyslipidemia, and cumulative risk scores and the lowest prevalence of dysphagia of the three clusters.

\section{Discussion}

The LCA of subacute ischemic stroke patients with moderate-to-severe OSA reveals three distinct clusters based on demographic data and medical comorbidities. Clusters 1 and 2 contained relatively older patients with differing predominant sexes and extent of comorbidities. Malepredominant Cluster 3 contained patients of relatively young age with the highest BMI and prevalence of comorbidities.

Phenotypes found by previous large cohort studies ${ }^{2-4}$ are not applicable to ischemic stroke patients with OSA. As the typical clinical manifestations of OSA, such as obesity and daytime sleepiness, are not obvious in postacute stroke patients, ${ }^{5,24}$ the prevalence of EDS did not differ significantly among the three clusters found in this study. Given the multifactorial etiology of post-stroke insomnia mentioned above, the prevalence of sedative usage also did not differ among the three clusters. Results of this study are consistent with previous studies ${ }^{8}$ of ischemic stroke patients with OSA which have suggested that EDS and insomnia are not major determinants in LCA.

Participants in Clusters 1 and 2 were older on average and the main difference between them was sex. The significantly higher prevalence of Af in female-predominant Cluster 1 supports previous studies of ischemic stroke patients that found Af was more prevalent in females. ${ }^{25}$ Increased AIx was found to be associated with new-onset Af and prevalence of $\mathrm{Af}^{26,27}$ A $10 \%$ absolute increase in AIx resulted in an increase in cardiovascular events of $26 \%$ and all-cause mortality of $38 \%{ }^{28}$ Our finding of a significantly higher AIx@75 and prevalence of Af in Cluster 1 patients as compared to age-matched patients in Cluster 2 is in agreement with these findings and suggests that patients in Cluster 1 may require more aggressive treatment for OSA and comorbidities compared to those in Cluster 2. Park et al found that the hypopnea index was a more important predictor of heart failure and coronary artery disease than the apnea index and that patients with hypopnea-predominant OSA had a significantly larger female to male ratio as compared to those with apneapredominant OSA. ${ }^{29}$ The findings that femalepredominant Cluster 1 had the highest hypopnea index of the three clusters and that Af is closely related to coronary artery disease and heart failure ${ }^{30}$ further support our inference based on elevated AIx@75 that the risk of cardiovascular events and mortality was higher in Cluster 1 patients compared to those in Cluster 2. Studies have found that non-anatomical pathogeneses of OSA cause dynamic obstruction of the airway and hypopnea, while anatomical pathogenesis of OSA causes static obstruction and apnea. ${ }^{29}$ Together with the findings that patients in Cluster 1 had the thinnest neck circumference, Cluster 1 patients may be more likely to have nonanatomic pathogeneses of OSA and are good candidates for alternative therapy along with standard continuous positive airway pressure treatment, as suggested by Eckert et al. ${ }^{31}$

In contrast to Cluster 1, patients in Clusters 2 and 3 were mainly male and the major difference between these two clusters was age. In addition, participants in Cluster 2 had the most normal BMI and lowest prevalence of HTN, and tended to have the lowest cumulative risk score for cardiovascular diseases and recurrent stroke prevalence of 
the three clusters, which supports previous findings that the mortality risk of OSA among men with an apneahypopnea index $>50$ decreased with increasing age. ${ }^{32}$ The mechanisms for this perplexing phenomenon are not well understood. With aging, the arousal threshold is reduced, sleep fragmentation is increased, and the level of nocturnal hypoxia is decreased for a given apneahypopnea index. ${ }^{33}$ Therefore, the elderly with OSA have less nocturnal intermittent hypoxia and its related consequences. ${ }^{33}$ Our findings that participants in Cluster 3 had better sleep efficiency and lower levels of minimal oxyhemoglobin saturation by pulse oximetry as compared to participants in Cluster 2 are in agreement with these results. Alternatively, the protective effect against mortality of increasing age might result from the fact that only patients who could successfully adapt to nocturnal respiratory events could survive into older age. ${ }^{32}$ Whether treatment for OSA provides clinical benefits for patients in Cluster 2 requires further study. However, Cluster 2 patients had the highest prevalence of depression. Given that post-stroke depression is not consistently associated with age and sex, ${ }^{34}$ neurocognitive dysfunction due to OSA might be more severe in patients in Cluster 2, a possibility that merits further study.

Patients in Cluster 3 were the youngest and had the highest BMI, neck circumference, BI, and cumulative risk score of all three clusters. The characteristics of participants in Cluster 3 are similar to typical OSA presentation, including being male, overweight and having a thick neck. ${ }^{35}$ Given that AIx increases with age, ${ }^{36}$ the nonsignificant difference in AIx@75 between patients in Cluster 3 and their older counterparts in Clusters 1 and 2 suggests that Cluster 3 has elevated AIx and increased risk of cardiovascular events and mortality. ${ }^{28}$ In contrast to Cluster 1, patients in Cluster 3 had the thickest necks and apnea-predominant OSA, and may have primarily anatomic contributors to the pathophysiology of OSA. Therefore, novel alternative therapies for OSA may be ineffective. That Cluster 3 had the highest BI is in agreement with previous observations that age independently and selectively influences stroke outcomes regarding the activities of daily living. ${ }^{37}$ That Cluster 3 also had the lowest rate of dysphagia is also consistent with previous findings that old age is an independent predictor of dysphagia, chest infection, or aspiration within the six months after a stroke. $^{38}$

This study has several limitations. Firstly, the study recruited a highly selective group of post-acute ischemic stroke patients who were admitted for inpatient rehabilitation with moderate-severe OSA. Therefore, our results cannot be extrapolated to ischemic stroke patients with mild OSA and to patients with mild or severe stroke severity who are not suitable for inpatient rehabilitation. However, the clinical impact of mild OSA on stroke recurrence, recovery and mortality seems to be unremarkable. Secondly, sleep disturbance was determined by whether or not patients were prescribed sedatives, rather than by subjective symptoms or objective measures. Given that environmental factors, such as residing in a rehabilitation ward, strongly influence sleep, inpatients commonly report subjective symptoms of sleep disturbance, so we selected severe sleep disturbance with sedative use as our variable in the LCA. Finally, future longitudinal research is suggested to validate the inferences of this cross-sectional analysis.

\section{Conclusions}

Ischemic stroke patients with moderate-to-severe OSA can be categorized into three clusters based on age, sex, and medical comorbidities. The results of LCA have clinical relevance and applicability by providing valuable information for risk stratification and treatment selection for OSA.

\section{Abbreviations}

OSA, obstructive sleep apnea; EDS, excessive daytime sleepiness; LCA, latent class analysis; BMI, body mass index; HTN, hypertension; BI, Barthel index.

\section{Author Contributions}

All authors met the following conditions 1, 2, 3, 4 and 5 .

1. Made a significant contribution to the work reported, whether that is in the conception, study design, execution, acquisition of data, analysis and interpretation, or in all these areas.

2. Have drafted or written, or substantially revised or critically reviewed the article.

3. Have agreed on the journal to which the article will be submitted.

4. Reviewed and agreed on all versions of the article before submission, during revision, the final version accepted for publication, and any significant changes introduced at the proofing stage.

5. Agree to take responsibility and be accountable for the contents of the article. 


\section{Funding}

Study funded by the Chang Gung Medical Research Council under Contract No. CMRPG2K0051.

\section{Disclosure}

On behalf of all authors, the corresponding author states that there is no conflict of interest.

\section{References}

1. Bassetti CLA, Randerath W, Vignatelli L, et al. EAN/ERS/ESO/ ESRS statement on the impact of sleep disorders on risk and outcome of stroke. Eur Respir J. 2020;55(4):1901104. doi:10.1183/ 13993003.01104-2019

2. Ye L, Pien GW, Ratcliffe SJ, et al. The different clinical faces of obstructive sleep apnoea: a cluster analysis. Eur Respir J. 2014;44 (6):1600-1607. doi:10.1183/09031936.00032314

3. Keenan BT, Kim J, Singh B, et al. Recognizable clinical subtypes of obstructive sleep apnea across international sleep centers: a cluster analysis. Sleep. 2018;41(3):zsx214. doi:10.1093/sleep/zsx214

4. Mazzotti DR, Keenan BT, Lim DC, Gottlieb DJ, Kim J, Pack AI. Symptom subtypes of obstructive sleep apnea predict incidence of cardiovascular outcomes. Am J Respir Crit Care Med. 2019;200 (4):493-506. doi:10.1164/rccm.201808-1509OC

5. McKee Z, Auckley DH. A sleeping beast: obstructive sleep apnea and stroke. Cleve Clin J Med. 2019;86(6):407-415. doi:10.3949/ ccjm.86a. 18033

6. Leppävuori A, Pohjasvaara T, Vataja R, Kaste M, Erkinjuntti T. Insomnia in ischemic stroke patients. Cerebrovasc Dis. 2002;14 (2):90-97. doi:10.1159/000064737

7. Bassetti CL. Sleep and stroke. Semin Neurol. 2005;25(1):19-32. doi:10.1055/s-2005-867073

8. Schutz SG, Lisabeth LD, Shafie-Khorassani F, et al. Clinical phenotypes of obstructive sleep apnea after ischemic stroke: a cluster analysis. Sleep Med. 2019;60:178-181. doi:10.1016/j. sleep.2019.04.004

9. Joint committee for guideline. 2016 Chinese guidelines for the management of dyslipidemia in adults. J Geriatr Cardiol. 2018;15 (1):1-29. doi:10.11909/j.issn.1671-5411.2018.01.011.

10. Johns MW. A new method for measuring daytime sleepiness: the Epworth sleepiness scale. Sleep. 1991;14(6):540-545. doi:10.1093/ sleep/14.6.540

11. Shah S, Vanclay F, Cooper B. Improving the sensitivity of the Barthel index for stroke rehabilitation. $J$ Clin Epidemiol. 1989;42 (8):703-709. doi:10.1016/0895-4356(89)90065-6

12. Newman AB, Spiekerman CF, Enright P, et al. Daytime sleepiness predicts mortality and cardiovascular disease in older adults. The cardiovascular health study research group. $J$ Am Geriatr Soc. 2000;48(2):115-123. doi:10.1111/j.1532-5415.2000.tb03901.x

13. Jaussent I, Empana JP, Ancelin ML, et al. Insomnia, daytime sleepiness and cardio-cerebrovascular diseases in the elderly: a 6-year prospective study. PLoS One. 2013;8(2):e56048. doi:10.1371/journal.pone. 0056048

14. Manea L, Gilbody S, McMillan D. A diagnostic meta-analysis of the Patient Health Questionnaire-9 (PHQ-9) algorithm scoring method as a screen for depression. Gen Hosp Psychiatry. 2015;37(1):67-75. doi:10.1016/j.genhosppsych.2014.09.009

15. de Man-van Ginkel J, Hafsteinsdottir TRNPD, Lindeman EMDPD, Burger HMDPD, Grobbee DMDPD, Schuurmans MRNPD. An efficient way to detect poststroke depression by subsequent administration of a 9-item and a 2-item patient health questionnaire. Stroke. 2012;43(3):854-856. doi:10.1161/STROKEAHA.111.640276
16. Daviglus ML, Talavera GA, Aviles-Santa ML, et al. Prevalence of major cardiovascular risk factors and cardiovascular diseases among Hispanic/Latino individuals of diverse backgrounds in the United States. JAMA. 2012;308(17):1775-1784. doi:10.1001/ jama.2012.14517

17. Berry R, Brooks R, Gamaldo C The AASM manual for the scoring of sleep and associated events: rules, terminology and technical specifications, version 2.0.3. American Academy of Sleep Medicine; 2014.

18. O'Rourke MF, Blazek JV, Morreels CL, Krovetz LJ. Pressure wave transmission along the human Aorta: changes with age and in arterial degenerative disease. Circ Res. 1968;23(4):567-579. doi:10.1161/01. RES.23.4.567

19. O'Rourke MF, Adji A. An updated clinical primer on large artery mechanics: implications of pulse waveform analysis and arterial tonometry. Curr Opin Cardiol. 2005;20(4):275-281. doi:10.1097/ 01.hco.0000166595.44711.6f

20. Chen CY, Chen CL, Yu CC. Obstructive sleep apnea is independently associated with arterial stiffness in ischemic stroke patients. $J$ Neurol. 2015;262(5):1247-1254. doi:10.1007/s00415-015-7699-2

21. Karamanoglu M, O’Rourke MF, Avolio AP, Kelly RP. An analysis of the relationship between central aortic and peripheral upper limb pressure waves in man. Eur Heart J. 1993;14(2):160-167. doi:10.1093/eurheartj/14.2.160

22. Safar ME, London GM; for The Clinical Committee of Arterial S, Function, on behalf of the Working Group on Vascular S, Function of the European Society of $\mathrm{H}$. Therapeutic studies and arterial stiffness in hypertension: recommendations of the European Society of Hypertension. J Hypertens. 2000;18(11):1527-1535. doi:10.1097/ 00004872-200018110-00001

23. Wilkinson IB, MacCallum H, Flint L, Cockcroft JR, Newby DE, Webb DJ. The influence of heart rate on augmentation index and central arterial pressure in humans. template. $J$ Physiol. 2000;525 (1):263-270. doi:10.1111/j.1469-7793.2000.t01-1-00263.x

24. Chan WB, Coutts SBM, Hanly PMD. Sleep apnea in patients with transient ischemic attack and minor stroke: opportunity for risk reduction of recurrent stroke? Stroke. 2010;41(12):2973-2975. doi:10.1161/STROKEAHA.110.596759

25. Friberg LP, Rosenqvist MP, Lindgren AP, Terent AP, Norrving BP, Asplund KP. High prevalence of atrial fibrillation among patients with ischemic stroke. Stroke. 2014;45(9):2599-2605. doi:10.1161/ STROKEAHA.114.006070

26. Shaikh AY, Wang N, Yin X, et al. Relations of Arterial Stiffness and brachial flow-mediated dilation with new-onset atrial fibrillation: the Framingham Heart Study. Hypertension. 2016;68(3):590-596. doi:10.1161/HYPERTENSIONAHA.116.07650

27. Cui R, Yamagishi K, Muraki I, et al. Association between markers of arterial stiffness and atrial fibrillation in the Circulatory Risk in Communities Study (CIRCS). Atherosclerosis. 2017;263:244-248. doi:10.1016/j.atherosclerosis.2017.06.918

28. Vlachopoulos C, Aznaouridis K, O'Rourke MF, Safar ME, Baou K, Stefanadis C. Prediction of cardiovascular events and all-cause mortality with central haemodynamics: a systematic review and meta-analysis. Eur Heart J. 2010;31(15):1865-1871. doi:10.1093/ eurheartj/ehq024

29. Park S, Shin B, Lee J-H, et al. Polysomnographic phenotype as a risk factor for cardiovascular diseases in patients with obstructive sleep apnea syndrome: a retrospective cohort study. J Thorac Dis. 2020;12 (3):907-915. doi:10.21037/jtd.2019.12.66

30. Staerk L, Sherer JA, Ko D, Benjamin EJ, Helm RH. Atrial fibrillation: epidemiology, pathophysiology, and clinical outcomes review. Circ Res. 2017;120(9):1501-1517. doi:10.1161/ CIRCRESAHA.117.309732

31. Eckert DJ, White DP, Jordan AS, Malhotra A, Wellman A. Defining phenotypic causes of obstructive sleep apnea. Identification of novel therapeutic targets. Am J Respir Crit Care Med. 2013;188 (8):996-1004. doi:10.1164/rccm.201303-04480C 
32. Lavie P, Lavie L, Herer P. All-cause mortality in males with sleep apnoea syndrome: declining mortality rates with age. Eur Respir J. 2005;25(3):514-520. doi:10.1183/09031936.05.00051504

33. Beaudin AE, Waltz X, Hanly PJ, Poulin MJ. Impact of obstructive sleep apnoea and intermittent hypoxia on cardiovascular and cerebrovascular regulation. Exp Physiol. 2017;102(7):743-763. doi:10.1113/ep086051

34. Robinson RG, Jorge RE. Post-stroke depression: a review. Am $J$ Psychiatry. 2016;173(3):221-231. doi:10.1176/appi.ajp.2015. 15030363

35. Victor LD. Obstructive sleep apnea. Am Fam Physician. 1999;60 (8):2279-2286.
36. Namasivayam M, Adji A, O'Rourke MF. Aortic augmentation index and aging: mathematical resolution of a physiological dilemma? Hypertension. 2010;56(1):e9-e10. doi:10.1161/HYPERTEN SIONAHA.110.153742

37. Nakayama H, Jørgensen H, Raaschou H, Olsen TS. The influence of age on stroke outcome. The Copenhagen Stroke Study. Stroke. 1994;25(4):808-813. doi:10.1161/01.STR.25.4.808

38. Mann G, Hankey GJ, Cameron D. Swallowing function after stroke: prognosis and prognostic factors at 6 months. Stroke. 1999;30 (4):744-748. doi:10.1161/01.STR.30.4.744

\section{Publish your work in this journal}

Nature and Science of Sleep is an international, peer-reviewed, open access journal covering all aspects of sleep science and sleep medicine, including the neurophysiology and functions of sleep, the genetics of sleep, sleep and society, biological rhythms, dreaming, sleep disorders and therapy, and strategies to optimize healthy sleep.
The manuscript management system is completely online and includes a very quick and fair peer-review system, which is all easy to use. Visit http://www.dovepress.com/testimonials.php to read real quotes from published authors. 